\title{
Exact controllability in projections of the bilinear Schrödinger equation*
}

\author{
Marco Caponigro ${ }^{\dagger} \quad$ Mario Sigalotti ${ }^{\ddagger \delta}$
}

May 18, 2018

\begin{abstract}
We consider the bilinear Schrödinger equation with discrete-spectrum drift. We show, for $n \in \mathbb{N}$ arbitrary, exact controllability in projections on the first $n$ given eigenstates. The controllability result relies on a generic controllability hypothesis on some associated finite-dimensional approximations. The method is based on Lie-algebraic control techniques applied to the finite-dimensional approximations coupled with classical topological arguments issuing from degree theory.
\end{abstract}

\section{Introduction}

In this paper we study the controllability problem for the multi-input Schrödinger equation

$$
i \frac{d \psi}{d t}(t)=\left(H_{0}+u_{1}(t) H_{1}+\ldots+u_{p}(t) H_{p}\right) \psi(t)
$$

where $H_{0}, \ldots, H_{p}$ are self-adjoint operators on a Hilbert space $\mathcal{H}$ and the drift Schrödinger operator $H_{0}$ (the internal Hamiltonian) has discrete spectrum. The control functions $u_{1}(\cdot), \ldots, u_{p}(\cdot)$, representing external fields, are real-valued and $\psi(\cdot)$ takes values in the unit sphere of $\mathcal{H}$.

In recent years there has been an increasing interest in studying the controllability of the bilinear Schrödinger equation (1), mainly due to its importance for many applications such as laser spectroscopy or quantum information. The problem concerns the design of control laws $\left(u_{1}, \ldots, u_{p}\right)$ steering the system from a given initial state to a pre-assigned final state in a given time.

The controllability of system (1) is a well-established topic when the state space $\mathcal{H}$ is finitedimensional (see for instance [15] and reference therein), thanks to general controllability methods for left-invariant control systems on compact Lie groups $([21,20,17,16])$.

We are interested here in the case in which $\mathcal{H}$ is infinite-dimensional. When the control operators $H_{1}, \ldots, H_{p}$ are bounded, it is known that the bilinear Schrödinger equation is not exactly controllable (see $[4,35]$ ). Hence, it is natural to look for weaker controllability properties such as approximate controllability or controllability between eigenstates of the Schödinger operator. In certain cases it is possible to prove exact controllability in suitable functional spaces on a real interval (see $[5,6$,

${ }^{*}$ This work was supported by the project DISQUO of the DEFI InFIniTI 2017 by CNRS and the QUACO project by ANR number 17-CE40-0007-01

${ }^{\dagger}$ Conservatoire National des Arts et Métiers, Équipe M2N, Paris, France (marco.caponigro@cnam.fr)

${ }^{\ddagger}$ Inria (mario.sigalotti@inria.fr)

§Sorbonne Université, Université Paris-Diderot SPC, CNRS, Inria, Laboratoire Jacques-Louis Lions, Paris, France 
27]). In $\mathbb{R}^{d}, d>1$, the exact description of the reachable set seems a difficult task. However, approximate controllability results have been obtained with different techniques: adiabatic control $([1,11])$, Lyapunov methods $([25,28,29,30])$, and Lie-algebraic methods $([14,8,9,13,10,7,22,31])$.

The Lie-algebraic approach developed in $[14,9,13,10]$ serves as basis for the analysis in this paper. The basic idea is to drive the system with control laws that are in resonance with spectral gaps of the internal Hamiltonian $H_{0}$. The resonances are used to identify finite-dimensional dynamics which can be tracked with arbitrary precision by the infinite-dimensional system. In [10] we used this reduced finite dimensional dynamics to introduce the Lie-Galerkin Control Condition (see Definition 4 below), which ensures approximate controllability of $(1)$ in the unit sphere of $\mathcal{H}$. The condition applies for very degenerate spectra of the internal Hamiltonian $H_{0}$ and also guarantees approximate operator controllability, approximate controllability in finer topologies, and tracking.

In this paper we go beyond approximate controllability and prove that the Lie-Galerkin Control Condition implies a stronger controllability property: exact controllability in projections. More precisely, our main result, Theorem 3 , states that given a Hilbert basis $\left(\phi_{k}\right)_{k \in \mathbb{N}}$ of $\mathcal{H}$ made of eigenvectors of $H_{0}$, for every given $\mathfrak{n} \in \mathbb{N}$, initial condition $\psi_{\text {in }} \in \mathcal{H}$ with $\left\|\psi_{\text {in }}\right\|=1$, and final condition $\psi_{\mathrm{f}} \in \mathcal{H}$ such that $\left\|\psi_{\mathrm{f}}\right\|=1$ with $\left\langle\psi_{\mathrm{f}}, \phi_{j}\right\rangle \neq 0$ for some $j>\mathfrak{n}$ there exists an admissible control $t \mapsto\left(u_{1}(t), \ldots, u_{p}(t)\right)$ such that the associated solution $t \mapsto \psi(t)$ of (1) with $\psi(0)=\psi_{\text {in }}$ satisfies $\left\langle\Upsilon_{T}^{u}\left(\psi_{\text {in }}\right), \phi_{j}\right\rangle=\left\langle\psi_{\mathrm{f}}, \phi_{j}\right\rangle$ for every $j=1, \ldots, \mathfrak{n}$. Exact controllability in projections guarantees, for example, that given any initial condition $\psi_{\text {in }}$ and any $n \in \mathbb{N}$, it is possible to steer in finite time $\psi_{\text {in }}$ to the orthogonal complement of $\operatorname{span}\left\{\phi_{1}, \ldots, \phi_{n}\right\}$. It also guarantees the possibility to implement exactly, in finite time, a Quantum Random Number Generation protocol (see e.g., [23, 19]) with an infinite dimensional system. In the simplest protocol, for instance, the initial state is steered to a final state who has a probability of $1 / 2$ to be measured in the ground state.

The idea of the proof of exact controllability in projections is the following. The Lie-Galerkin Control Condition provides controllability for any fixed finite-dimensional approximation while avoiding the transfer of population to higher energy levels for higher-dimensional approximations. This yields estimates on the difference between the dynamics of the finite-dimensional approximation and the original infinite-dimensional system. This fact combined with the continuity of the input-output mapping (see Assumption $(\mathbb{A} 5)$ ) and a topological degree argument ensures exact controllability in projections.

The hypothesis that the final condition $\psi_{\mathrm{f}}$ satisfies $\left\langle\psi_{\mathrm{f}}, \phi_{j}\right\rangle \neq 0$ for some $j>\mathfrak{n}$ cannot be removed. Since, as we have already recalled, one cannot expect exact controllability tout court if the control operators $H_{1}, \ldots, H_{p}$ are regular (e.g., continuous). The regularity of the control operators, and as a consequence of the input-output mapping, is therefore an obstruction for the exact controllability while, on the other hand, continuity of the input-output mapping is an assumption needed for the application of the topological degree methods used in the proof of Theorem 3 below. In this sense the controllability in projections is the strongest general controllability property that one may expect in the framework of bounded control potentials.

\section{Framework and main result}

Let $p \in \mathbb{N}, \delta>0$, and $U=U_{1} \times \cdots \times U_{p}$ with either $U_{j}=[0, \delta]$ or $U_{j}=[-\delta, \delta]$. For simplicity of notation we consider the bilinear control systems obtained by replacing the operators in (1) by $A=-i H_{0}$ and $B_{j}=-i H_{j}, j=1, \ldots, p$. This leads to the following definition. 
Definition 1. Let $\mathcal{H}$ be an infinite-dimensional Hilbert space with scalar product $\langle\cdot, \cdot\rangle$ and $A, B_{1}, \ldots, B_{p}$ be (possibly unbounded) skew-adjoint operators on $\mathcal{H}$, with domains $D(A), D\left(B_{1}\right), \ldots, D\left(B_{p}\right)$. Let us introduce the controlled equation

$$
\frac{d \psi}{d t}(t)=\left(A+u_{1}(t) B_{1}+\cdots+u_{p}(t) B_{p}\right) \psi(t), \quad u(t) \in U .
$$

We say that $A$ satisfies $(\mathbb{A} 1)$ if the following assumption holds true.

(A1) $A$ has discrete spectrum with infinitely many distinct eigenvalues (possibly degenerate).

Note that $(\mathbb{A} 1)$ is true whenever $A$ has compact resolvent. Denote by $\Phi$ a Hilbert basis $\left(\phi_{k}\right)_{k \in \mathbb{N}}$ of $\mathcal{H}$ made of eigenvectors of $A$ associated with the family of eigenvalues $\left(i \lambda_{k}\right)_{k \in \mathbb{N}}$ and let $\mathcal{L}$ be the set of finite linear combinations of eigenstates, that is,

$$
\mathcal{L}=\bigcup_{k \in \mathbb{N}} \operatorname{span}\left\{\phi_{1}, \ldots, \phi_{k}\right\}
$$

We consider the following assumptions on the regularity of the control operators $B_{1}, \ldots, B_{p}$ :

$(\mathbb{A} 2) \phi_{k} \in D\left(B_{j}\right)$ for every $k \in \mathbb{N}, j=1, \ldots, p$;

(A3) $A+u_{1} B_{1}+\cdots+u_{p} B_{p}: \mathcal{L} \rightarrow \mathcal{H}$ is essentially skew-adjoint for every $u \in U$.

When $\left(A, B_{1}, \ldots, B_{p}, \Phi, U\right)$ satisfies $(\mathbb{A} 1)-(\mathbb{A} 2)-(\mathbb{A} 3)$ we define the solution of $(2)$ as follows.

Definition 2. We say that $u \in L^{\infty}\left([0, T], \mathbb{R}^{p}\right)$ is admissible for $(2)$ if $u(t) \in U$ for almost every $t \in[0, T]$ and, for every $\psi_{0} \in \mathcal{H}$, there exists $\psi:[0, T] \rightarrow \mathcal{H}$ such that $\psi(0)=\psi_{0}$ the function $t \mapsto\left\langle\psi(t), \phi_{k}\right\rangle$ is absolutely continuous for every $k \in \mathbb{N}$ and satisfies

$$
\frac{d}{d t}\left\langle\phi_{k}, \psi(t)\right\rangle=-\left\langle\left(A+u_{1}(t) B_{1}+\cdots+u_{p}(t) B_{p}\right) \phi_{k}, \psi(t)\right\rangle,
$$

for almost every $t \in[0, T]$. The function $t \mapsto \psi(t)$ is called solution of $(2)$ with initial condition $\psi_{0} \in \mathcal{H}$ associated with the control $u$.

Assumption (A3) implies that the norm of the solutions given by Definition 2 is constant along the evolution. In particular it guarantees the uniqueness of solutions. We can therefore define the unitary propagator of (2), denoted by $\Upsilon_{t}^{u}$, as follows.

Definition 3. Let $u:[0, T] \rightarrow U$ be admissible for (2). The mapping $[0, T] \ni t \mapsto \Upsilon_{t}^{u}$ where $\Upsilon_{t}^{u} \psi_{0}$ is the evaluation at time $t$ of the solution of (2) with initial condition $\psi_{0} \in \mathcal{H}$ associated with $u$, is called propagator of (2).

Let $\left(A, B_{1}, \ldots, B_{p}, \Phi, U\right)$ satisfy $(\mathbb{A} 1)-(\mathbb{A} 2)-(\mathbb{A} 3)$ and let $\mathcal{U} \subset L^{\infty}([0, \infty), U)$. We say that $\left(A, B_{1}, \ldots, B_{p}, \Phi, U, \mathcal{U}\right)$ satisfies $(\mathbb{A} 4)$ if

(A4) every $u \in \mathcal{U}$ is admissible.

Assumption $(\mathbb{A} 4)$ holds true for the class of piecewise constant controls and, under suitable regularity conditions, for the class of smooth controls as detailed in the following two remarks. 
Remark 1. Let $\left(A, B_{1}, \ldots, B_{p}, \Phi, U\right)$ satisfy $(\mathbb{A} 1)-(\mathbb{A} 2)-(\mathbb{A} 3)$ and let $u(\cdot)=\left(u_{1}(\cdot), \ldots, u_{p}(\cdot)\right)$ be a $p$-tuple of piecewise constant controls on $[0, T]$ with value in $U$. Then $u$ is admissible for $(2)$ and the propagator is given by

$$
\Upsilon_{t}^{u}=e^{\left(t-\sum_{l=1}^{j-1} t_{l}\right)\left(A+u_{1}^{(j)} B_{1}+\cdots+u_{p}^{(j)} B_{p}\right)} \circ \cdots \circ e^{t_{1}\left(A+u_{1}^{(1)} B_{1}+\cdots+u_{p}^{(1)} B_{p}\right)},
$$

where $\sum_{l=1}^{j-1} t_{l} \leq t<\sum_{l=1}^{j} t_{l}$ and $u(\tau)=\left(u_{1}^{(j)}, \ldots, u_{p}^{(j)}\right) \in U$ if $\sum_{l=1}^{j-1} t_{l} \leq \tau<\sum_{l=1}^{j} t_{l}$. Indeed, since

$$
\left\langle\phi_{n}, e^{t\left(A+u_{1} B_{1}+\cdots+u_{p} B_{p}\right)} \psi_{0}\right\rangle=\left\langle e^{-t\left(A+u_{1} B_{1}+\cdots+u_{p} B_{p}\right)} \phi_{n}, \psi_{0}\right\rangle,
$$

then $t \mapsto \psi(t)=\Upsilon_{t}^{u} \psi_{0}$ satisfies (3) for almost every $t \in[0, T]$.

Remark 2. Let $u \in C^{1}([0, T], U)$ and $B_{1}, \ldots, B_{p}$ be $A$-bounded with $A$-bound smaller than $1 / \delta$ in the sense of $[32$, Section X.2], i.e.

- $D\left(B_{j}\right) \supset D(A)$,

- there exists $a<1 / \delta$ and $b \in \mathbb{R}$ such that for all $\phi \in D(A)$ one has

$$
\left\|B_{j} \phi\right\| \leq a\|A \phi\|+b\|\phi\|
$$

for every $j=1, \ldots, p$. Then by the Kato-Rellich theorem ([32, Theorem X.12]) and [32, Theorem X.70] $u$ is admissible for (2). If, moreover, $\psi_{0} \in D(A)$ then $t \mapsto \Upsilon_{t}^{u} \psi_{0}$ satisfies (2) for every $t \in[0, T]$.

We say that $\left(A, B_{1}, \ldots, B_{p}, \Phi, U, \mathcal{U}\right)$ satisfies $(\mathbb{A})$ if it satisfies $(\mathbb{A} 1)-(\mathbb{A} 2)-(\mathbb{A} 3)-(\mathbb{A} 4)$ and the following additional assumption.

(A5) The input-output mapping is continuous in the sense that if $\left(u_{n}\right)_{n \in \mathbb{N}} \subset \mathcal{U}$ and $u \in \mathcal{U}$ are such that $u_{n} \rightarrow u$ in $L^{1}([0, T])$ as $n \rightarrow \infty$ then $\Upsilon_{t}^{u_{n}} \phi$ tends to $\Upsilon_{t}^{u} \phi$ in $\mathcal{H}$ uniformly with respect to $t \in[0, T]$ as $n \rightarrow \infty$ for every $\phi \in \mathcal{H}$.

Remark 3. In the case in which $A$ satisfies $(\mathbb{A} 1)$ and $B_{1}, \ldots, B_{p}$ are bounded operators, assumptions $(\mathbb{A} 2),(\mathbb{A} 3)$ are clearly verified. Assumption $(\mathbb{A} 5)$ is the consequence of $[4$, Theorem 3.6]. More general conditions on $B_{1}, \ldots, B_{p}$ ensuring that $(\mathbb{A} 5)$ holds true can be found for instance in [12, Section 2.3].

For $n \in \mathbb{N}$ we denote by $\Pi_{n}$ the projection of $\mathcal{H}$ on the span of the first $n$ eigenvectors of $A$, namely

$$
\begin{array}{rlc}
\Pi_{n}: \mathcal{H} & \rightarrow & \mathcal{H} \\
\psi & \mapsto & \sum_{k=1}^{n}\left\langle\phi_{k}, \psi\right\rangle \phi_{k} .
\end{array}
$$

When it does not create ambiguities we identify $\operatorname{Im}\left(\Pi_{n}\right)=\operatorname{span}\left\{\phi_{1}, \ldots, \phi_{n}\right\}$ with $\mathbb{C}^{n}$. Given a linear operator $Q$ on $\mathcal{H}$ we identify the linear operator $\pi_{n} Q \pi_{n}$ preserving $\operatorname{span}\left\{\phi_{1}, \ldots, \phi_{n}\right\}$ with its $n \times n$ complex matrix representation with respect to the basis $\left(\phi_{1}, \ldots, \phi_{n}\right)$. We define

$$
A^{(n)}=\Pi_{n} A \Pi_{n} \quad \text { and } \quad B_{j}^{(n)}=\Pi_{n} B_{j} \Pi_{n},
$$

for every $j=1, \ldots, p$.

Let us introduce the set $\Sigma_{n}$ of spectral gaps associated with the first $n$ eigenvalues of $A$ as

$$
\Sigma_{n}=\left\{\left|\lambda_{l}-\lambda_{k}\right| \mid l, k=1, \ldots, n\right\} .
$$


For every $\sigma \geq 0$, every $m \in \mathbb{N}$, and every $m \times m$ matrix $M$, let

$$
\mathcal{E}_{\sigma}(M)=\left(M_{l, k} \delta_{\sigma,\left|\lambda_{l}-\lambda_{k}\right|}\right)_{l, k=1}^{m},
$$

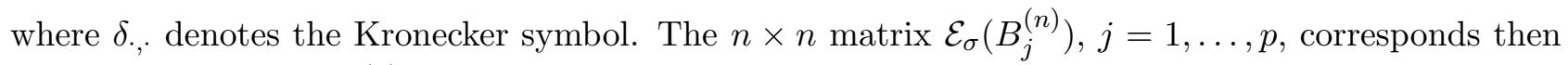
to the "selection" in $B_{j}^{(n)}$ of the spectral gap $\sigma$ : the $(l, k)$-elements such that $\left|\lambda_{l}-\lambda_{k}\right| \neq \sigma$ are set to 0 .

Define

$$
\Xi_{n}=\left\{(\sigma, j) \in \Sigma_{n} \times\{1, \ldots, p\} \mid\left(B_{j}\right)_{k, l} \delta_{\sigma,\left|\lambda_{l}-\lambda_{k}\right|}=0 \text {, for every } k=1, \ldots, n \text { and } l>n\right\} .
$$

The set $\Xi_{n}$ can be seen as follows: If $(\sigma, j) \in \Xi_{n}$ then the matrix $M=\mathcal{E}_{\sigma}\left(B_{j}^{(n)}\right)$ is such that

$$
\mathcal{E}_{\sigma}\left(B_{j}^{(N)}\right)=\left(\begin{array}{c|c}
M & 0 \\
\hline 0 & *
\end{array}\right) \text { for every } N>n
$$

In particular $\operatorname{span}\left\{\phi_{1}, \ldots, \phi_{n}\right\}$ is invariant for the evolution of $\mathcal{E}_{\sigma}\left(B_{j}^{(N)}\right)$ for every $N>n$. The spectral gaps $\sigma \in \Xi_{n}$ are, therefore, those for which the selections $\mathcal{E}_{\sigma}\left(B_{j}^{(n)}\right)$ define finite dimensional dynamics of order $n$ "decoupled" from the infinite dimensional evolution.

The main assumption of this paper, introduced in the definition below, is a Lie-algebraic condition on the set of the decoupled selections associated with the spectral gaps in $\Xi_{n}$.

Definition 4 ([10]). For every $n \in \mathbb{N}$ define

$$
\begin{aligned}
\mathcal{M}_{n}=\left\{A^{(n)}\right\} \cup\left\{\mathcal{E}_{\sigma}\left(B_{j}^{(n)}\right) \mid(\sigma, j) \in \Xi_{n} \text { and } j \text { is such that }(0, j) \in \Xi_{n}\right\} \\
\cup\left\{\mathcal{E}_{\sigma}\left(B_{j}^{(n)}\right) \mid(\sigma, j) \in \Xi_{n}, \sigma \neq 0, U_{j}=[-\delta, \delta]\right\} .
\end{aligned}
$$

We say that the Lie-Galerkin Control Condition holds if for every $n_{0} \in \mathbb{N}$ there exists $n>n_{0}$ such that

$$
\operatorname{Lie} \mathcal{M}_{n} \supseteq \mathfrak{s u}(n) .
$$

The Lie-Galerkin Control Condition is a sufficient condition for approximate controllability as stated in Theorem 1 below.

Definition 5. Let $\left(A, B_{1}, \ldots, B_{p}, \Phi, U, \mathcal{U}\right)$ satisfy $(\mathbb{A} 1)-(\mathbb{A} 2)-(\mathbb{A} 3)-(\mathbb{A} 4)$. We say that $(2)$ is approximately controllable by means of controls in $\mathcal{U}$ if for every $\psi_{0}, \psi_{1}$ in the unit sphere of $\mathcal{H}$ and every $\varepsilon>0$ there exists $u \in \mathcal{U}$ and $T>0$ such that $\left\|\psi_{1}-\Upsilon_{T}^{u}\left(\psi_{0}\right)\right\|<\varepsilon$.

Theorem 1 ([10, Theorem 2.6]). Let $\left(A, B_{1}, \ldots, B_{p}, \Phi, U\right)$ satisfy $(\mathbb{A} 1)-(\mathbb{A} 2)-(\mathbb{A} 3)$. If the LieGalerkin Control Condition holds then system (2) is approximately controllable by means of piecewise constant controls.

Theorem 1 can be extended to the class of smooth controls as stated in Theorem 2 below. The proof of this result is given in Section 4.

Theorem 2. Let $\mathcal{U}$ be the set of $C^{\infty}$ functions with values in $U$ and $\left(A, B_{1}, \ldots, B_{p}, \Phi, U, \mathcal{U}\right)$ satisfy $(\mathbb{A} 1)-(\mathbb{A} 2)-(\mathbb{A} 3)-(\mathbb{A} 4)$. If the Lie-Galerkin Control Condition holds then system (2) is approximately controllable by means of controls in $\mathcal{U}$. 
Our main result is the approximate controllability in projections as stated below. Theorem 2 is one of the main tools in its proof.

Theorem 3. Let $\mathcal{U}$ be either the set of piecewise constant functions with values in $U$ or the set of $C^{\infty}$ functions with values in $U$. Let $\left(A, B_{1}, \ldots, B_{p}, \Phi, U, \mathcal{U}\right)$ satisfy $(\mathbb{A})$. Assume that the Lie-Galerkin Control Condition holds. Then for every $\mathfrak{n} \in \mathbb{N}, \varepsilon>0$, for every initial condition $\psi_{\text {in }} \in \mathcal{H}$ with $\left\|\psi_{\mathrm{in}}\right\|=1$ and every final condition $\psi_{\mathrm{f}} \in \mathcal{H}$ such that $\left\|\psi_{\mathrm{f}}\right\|=1$ and $\left\|\Pi_{\mathfrak{n}}\left(\psi_{\mathrm{f}}\right)\right\|<1$, there exists $u:[0, T] \rightarrow U, u \in \mathcal{U}$ such that

$$
\Pi_{\mathfrak{n}}\left(\Upsilon_{T}^{u}\left(\psi_{\mathrm{in}}\right)\right)=\Pi_{\mathfrak{n}}\left(\psi_{\mathrm{f}}\right) \quad \text { and } \quad\left\|\Upsilon_{T}^{u}\left(\psi_{\mathrm{in}}\right)-\psi_{\mathrm{f}}\right\|<\varepsilon
$$

\subsection{The Lie-Galerkin Control Condition in examples}

\subsubsection{Non-resonant chain of connectedness}

An example of easily verifiable condition in the case of single-input systems which implies the LieGalerkin Control Condition is given by the existence of a non-resonant chain of connectedness, as we recall below. Let $p=1$ and call, for simplicity, $B=B_{1}$. Let $(A, B, \Phi, U)$ satisfy assumption $(\mathbb{A} 1)-(\mathbb{A} 2)-(\mathbb{A} 3)$.

Definition 6 ([9]). A subset $S$ of $\mathbb{N}^{2}$ couples two levels $l, k$ in $\mathbb{N}$, if there exists a finite sequence $\left(\left(s_{1}^{1}, s_{2}^{1}\right), \ldots,\left(s_{1}^{q}, s_{2}^{q}\right)\right)$ in $S$ such that

(i) $s_{1}^{1}=l$ and $s_{2}^{q}=k$;

(ii) $s_{2}^{j}=s_{1}^{j+1}$ for every $1 \leq j \leq q-1$;

(iii) $\left\langle\phi_{s_{1}^{j}}, B \phi_{s_{2}^{j}}\right\rangle \neq 0$ for $1 \leq j \leq q$.

$S$ is called a connectedness chain for $(A, B, \Phi, U)$ if $S$ couples every pair of levels in $\mathbb{N}$.

A connectedness chain is said to be non-resonant if for every $\left(s_{1}, s_{2}\right)$ in $S,\left|\lambda_{s_{1}}-\lambda_{s_{2}}\right| \neq\left|\lambda_{t_{1}}-\lambda_{t_{2}}\right|$ for every $\left(t_{1}, t_{2}\right)$ in $\mathbb{N}^{2} \backslash\left\{\left(s_{1}, s_{2}\right),\left(s_{2}, s_{1}\right)\right\}$ such that $\left\langle\phi_{t_{2}}, B \phi_{t_{1}}\right\rangle \neq 0$.

Proposition 4. Assume that $\left\langle\phi_{l}, B \phi_{k}\right\rangle=0$ whenever $l \neq k$ and $\lambda_{l}=\lambda_{k}$. If $(A, B, \Phi, U)$ admits a non-resonant connectedness chain then the Lie-Galerkin Control Condition holds.

Proof. The first assumption in the statement of the proposition implies that $0 \in \Xi_{n}$ for every $n$. The non-resonance condition on the eigenvalues implies that, for every $n, \Xi_{n}=\Sigma_{n}$. Hence

$$
\mathcal{M}_{n}=\left\{A^{(n)}\right\} \cup\left\{\mathcal{E}_{\sigma}\left(B^{(n)}\right) \mid \sigma \in \Sigma_{n}\right\} .
$$

Since, moreover, $\mathcal{E}_{\left|\lambda_{l}-\lambda_{k}\right|}\left(B^{(n)}\right)$ has two nonzero entries if $\left\langle\phi_{l}, B \phi_{k}\right\rangle \neq 0$, the Lie-Galerkin Control Condition follows easily from the existence of a connectedness chain (see, for instance the proof of $[9$, Proposition 3.1] for details).

Remark 4. As a consequence of Proposition 4, the Lie-Galerkin Control Condition is satisfied by a generic single-input bilinear Schrödinger equation

$$
i \dot{\psi}=(-\Delta+V) \psi+u W \psi
$$

on $\Omega$ bounded domain of $\mathbb{R}^{N}$ and $V$ and $W$ are smooth functions from $\Omega \rightarrow \mathbb{R}^{N}$, see $[24$, Theorem $3.4]$. 


\subsubsection{Rotating bipolar molecule}

Consider the control of the orientation of a rigid bipolar molecule in $\mathbb{R}^{3}$ modeled by the following Schrödinger equation on the unit sphere $S^{2}$ :

$$
\begin{aligned}
i \frac{\partial \psi(\theta, \varphi, t)}{\partial t}= & -\Delta \psi(\theta, \varphi, t)+ \\
& +\left(u_{1}(t) \sin \theta \cos \varphi+u_{2}(t) \sin \theta \sin \varphi+u_{3}(t) \cos \theta\right) \psi(\theta, \varphi, t),
\end{aligned}
$$

where $\theta, \varphi$ are the spherical coordinates, which are related to the Euclidean coordinates through the identities

$$
x=\sin \theta \cos \varphi, \quad y=\sin \theta \sin \varphi, \quad z=\cos \theta,
$$

while $\Delta$ is the Laplace-Beltrami operator on the sphere $S^{2}$ (called in this context the angular momentum operator), i.e.,

$$
\Delta=\frac{1}{\sin \theta} \frac{\partial}{\partial \theta}\left(\sin \theta \frac{\partial}{\partial \theta}\right)+\frac{1}{\sin ^{2} \theta} \frac{\partial^{2}}{\partial \varphi^{2}}
$$

The wavefunction $\psi$ evolves in the unit sphere $\mathcal{S}$ of $\mathcal{H}=L^{2}\left(S^{2}, \mathbb{C}\right)$. A basis of eigenvectors of the Laplace-Beltrami operator $\Delta$ is given by the spherical harmonics $Y_{\ell}^{m}(\theta, \varphi)$, which satisfy

$$
\Delta Y_{\ell}^{m}(\theta, \varphi)=-\ell(\ell+1) Y_{\ell}^{m}(\theta, \varphi)
$$

The spectrum of $A=i \Delta$ is $\{-i \ell(\ell+1) \mid \ell \in \mathbb{N}\}$. Each eigenvalue $-i \ell(\ell+1)$ is of finite multiplicity $2 \ell+1$. The control operators $B_{1}, B_{2}, B_{3}$ are the multiplication operators by $-i \cos \varphi \sin \theta,-i \sin \varphi \sin \theta$, $-i \cos \theta$, respectively, which are bounded.

For symmetry reasons, the system is not controllable if one the three control is constantly switched off. In particular, the argument of Proposition 4 does not apply directly.

The systems satisfies condition $(\mathbb{A} 1)-(\mathbb{A} 2)-(\mathbb{A} 3)$. Assumptions $(\mathbb{A} 4)$ and $(\mathbb{A} 5)$, for piecewise constant or $C^{1}$ smooth controls, follow from Remarks 1, 2, and 3. Equation (5) satisfies the LieGalerkin Control Condition as proved in [10, Lemma 3.2].

\subsubsection{Quantum harmonic oscillator}

The quantum harmonic oscillator is among the most important examples of quantum systems. Consider its controlled version (see, for instance, [26]), in which $\mathcal{H}=L^{2}(\mathbb{R}, \mathbb{C}$ ) and the equation (2) reads

$$
i \frac{\partial \psi}{\partial t}(x, t)=\frac{1}{2}\left(-\Delta+x^{2}\right) \psi(x, t)+u(t) x \psi(x, t) .
$$

A Hilbert basis of $\mathcal{H}$ made of eigenvectors of $A=-\frac{i}{2}\left(-\Delta+x^{2}\right)$ is given by the Hermite functions $\left(\phi_{n}\right)_{n \in \mathbb{N}}$, associated with the sequence $\left(-i \lambda_{n}\right)_{n \in \mathbb{N}}$ of eigenvalues, where $\lambda_{n}=n-1 / 2$. In the basis $\left(\phi_{n}\right)_{n \in \mathbb{N}}, B_{1}=B=-i x$ is tri-diagonal since

$$
\left\langle\phi_{j}, B \phi_{k}\right\rangle= \begin{cases}i \sqrt{k-1} & \text { if } j=k-1 \\ i \sqrt{k} & \text { if } j=k+1 \\ 0 & \text { otherwise }\end{cases}
$$

This system is known to be non-controllable, neither exactly nor approximately as proved in [26], while its Galerkin approximations of every order are [33].

In this example, for every $n, \Sigma_{n}=\{0,1,2, \ldots, n\}, \Xi_{n}=\Sigma_{n} \backslash\{1\}$ and $\mathcal{E}_{\sigma}\left(B^{(n)}\right)$ is the zero matrix for every $\sigma \in \Xi_{n}$. Hence the Lie-Galerkin Control Condition is not satisfied. 


\section{$2.2 \quad$ Strategy of the proof}

The strategy of the proof of Theorem 3 splits into two main arguments. The first argument is used to formalize the decoupling which we mentioned as the intuition behind the definition of the set $\mathcal{M}_{n}$. More precisely, for every $n \in \mathbb{N}$, we show that there exists a family $\mathcal{W}_{n}$ of $n \times n$ matrices having the same Lie algebra as $\mathcal{M}_{n}$, which defines a control system in $\mathbb{C}^{n}$ whose trajectories can be approximated arbitrarily well by the projection $\Pi_{n}$ of trajectories of (2). The second argument goes as follows: if $n$ is such that the Lie algebra generated by $\mathcal{W}_{n}$ (or, equivalently, by $\mathcal{M}_{n}$ ) is $\operatorname{su}(n)$, then for every point of the unit sphere of $\mathbb{C}^{n}$ there exists a control law at which the endpoint map of the control system defined by $\mathcal{W}_{n}$ is a submersion. Then, by structural stability of submersions, it is possible to deduce exact controllability in projection of (2).

The first of the two arguments mentioned above spans Sections 3 and 4 and allows, in particular, to provide a proof of Theorem 2. It consists in three steps: an averaging result used to compare (up to phases) the flow of a matrix of $\mathcal{W}_{n}$ with a trajectory of (2) corresponding to a control in resonance with one of the frequencies in $\Xi_{n}$ (see Lemma 6), a construction allowing to concatenate the averaging arbitrarily many times (see Proposition 8), and a concluding phase tuning step (see Lemma 9).

\section{Finite-dimensional approximations of infinite-dimensional propa- gators}

In this section we introduce an auxiliary control system whose solutions are, up to phases, trajectories of (2), as showed in Lemma 5 below.

For $t, u_{1}, \ldots, u_{p} \in \mathbb{R}$ set $\Theta(t, u)=\Theta\left(t, u_{1}, \ldots, u_{p}\right)=e^{-t A}\left(u_{1} B_{1}+\cdots+u_{p} B_{p}\right) e^{t A}$, which is a linear operator from $\mathcal{L}$ to $\mathcal{H}$. Note that

$$
\Theta\left(t, u_{1}, \ldots, u_{p}\right)_{j k}=\left\langle\phi_{k}, \Theta\left(t, u_{1}, \ldots, u_{p}\right) \phi_{j}\right\rangle=e^{i\left(\lambda_{k}-\lambda_{j}\right) t}\left(u_{1}\left(B_{1}\right)_{j k}+\cdots+u_{p}\left(B_{p}\right)_{j k}\right) .
$$

Consider the nonautonomous control system

$$
\dot{y}(t)=\Theta\left(t, u_{1}(t), \ldots, u_{p}(t)\right) y(t) .
$$

Admissible solutions of (6) are, as in Definition 2, absolutely continuous functions $y:[0, T] \rightarrow \mathcal{H}$ satisfying

$$
\frac{d}{d t}\left\langle\phi_{n}, y(t)\right\rangle=-\left\langle\Theta\left(t, u_{1}(t), \ldots, u_{p}(t)\right) \phi_{n}, y(t)\right\rangle,
$$

for any $n \in \mathbb{N}$ and for almost every $t \in[0, T]$.

Lemma 5. Let $u \in \mathcal{U}$. Then $u$ is admissible for (6) and the corresponding admissible solution $y:[0, T] \rightarrow \mathcal{H}$ associated with the initial condition $y(0)$ satisfies

$$
\Upsilon_{t}^{u}(y(0))=e^{t A} y(t)
$$

for every $t \in[0, T]$.

Proof. The function

$$
y(t)=e^{-t A} \Upsilon_{t}^{u}(y(0))
$$


satisfies

$$
\begin{aligned}
\frac{d}{d t}\left\langle\phi_{n}, y(t)\right\rangle & =\frac{d}{d t} e^{i t \lambda_{n}}\left\langle\phi_{n}, \Upsilon_{t}^{u}(y(0))\right\rangle \\
& =i \lambda_{n} e^{i t \lambda_{n}}\left\langle\phi_{n}, \Upsilon_{t}^{u}(y(0))\right\rangle+e^{i t \lambda_{n}} \frac{d}{d t}\left\langle\phi_{n}, \Upsilon_{t}^{u}(y(0))\right\rangle \\
& =i \lambda_{n} e^{i t \lambda_{n}}\left\langle\phi_{n}, \Upsilon_{t}^{u}(y(0))\right\rangle-e^{i t \lambda_{n}}\left\langle\left(A+u_{1}(t) B_{1}+\cdots+u_{p}(t) B_{p}\right) \phi_{n}, \Upsilon_{t}^{u}(y(0))\right\rangle \\
& =-e^{i t \lambda_{n}}\left\langle\left(u_{1}(t) B_{1}+\cdots+u_{p}(t) B_{p}\right) \phi_{n}, e^{t A} y(t)\right\rangle \\
& =-\left\langle\Theta\left(t, u_{1}(t), \ldots, u_{p}(t)\right) \phi_{n}, y(t)\right\rangle
\end{aligned}
$$

for every $n \in \mathbb{N}$ and for almost every $t \in[0, T]$.

The following lemma is inspired by [13, Theorem 1]. Here we denote by $\|\cdot\|_{L(E, \mathcal{H})}$ the norm of linear operators from a subspace $E$ of $\mathcal{H}$ to $\mathcal{H}$.

Lemma 6. Let $n \in \mathbb{N}, \sigma>0, j \in\{1, \ldots, p\}, \nu_{0}$ and $\nu_{1}$ in $\mathbb{R}$, and $a, b \in \mathbb{R}$ such that $0<a<b$. For every $N>n$ let $\omega^{N}=(\underbrace{0, \ldots, 0}_{j-1}, v^{N}, \underbrace{0, \ldots, 0}_{p-j}): \mathbb{R} \rightarrow \mathbb{R}^{p}$ be a periodic function of period $T=2 \pi / \sigma$, such that

$$
\int_{0}^{T} v^{N}(t) d t=\nu_{0}, \quad \int_{0}^{T} v^{N}(t) e^{i \sigma t} d t=\nu_{1}
$$

and

$$
\int_{0}^{T} v^{N}(t) e^{i m \sigma t} d t=0, \quad \text { for every } m \geq 2 \text { such that } m \sigma \in \Sigma_{N} .
$$

Assume that one of the following three conditions is satisfied: $(i)(\sigma, j)$ and $(0, j)$ are in $\Xi_{n}$; (ii) $(\sigma, j) \in \Xi_{n}$ and $\nu_{0}=0 ;($ iii $)(0, j) \in \Xi_{n}$ and $\nu_{1}=0$. Assume, moreover, that $\omega^{N} / K$ is admissible for every $N \in \mathbb{N}$ and every $K \in \mathbb{N}$ large enough. Then

$$
\lim _{N \rightarrow \infty} \lim _{K \rightarrow \infty}\left\|\Upsilon_{K T}^{\tau \omega^{N} / K}-e^{K T A} \exp \left(\tau\left(\nu_{0} \mathcal{E}_{0}\left(B_{j}^{(n)}\right)+\nu_{1} \mathcal{E}_{\sigma}\left(B_{j}^{(n)}\right)\right)\right)\right\|_{L\left(\Pi_{n}(\mathcal{H}), \mathcal{H}\right)}=0
$$

uniformly with respect to $\tau \in[a, b]$, where $\exp \left(\tau\left(\nu_{0} \mathcal{E}_{0}\left(B_{j}^{(n)}\right)+\nu_{1} \mathcal{E}_{\sigma}\left(B_{j}^{(n)}\right)\right)\right): \mathbb{C}^{n} \rightarrow \mathbb{C}^{n}$ is identified with an operator on $\mathcal{H}$.

Proof. Step 1. Fix, for now, $N>n$ and consider the Galerkin approximation of order $N$ of system (6), namely the system associated with $\Theta^{(N)}(t, u)=\Pi_{N} \Theta(t, u) \Pi_{N}$. Then, for every $s \leq t$ and $\tau \in[a, b]$,

$$
\begin{aligned}
\overrightarrow{\exp } \int_{K s}^{K t} \Theta^{(N)}\left(r, \frac{\tau \omega^{N}(r)}{K}\right) d r & =\overrightarrow{\exp } \int_{s}^{t} \Theta^{(N)}\left(K r, \tau \omega^{N}(K r)\right) d r \\
& =\overrightarrow{\exp } \int_{s}^{t} e^{-r K A^{(N)}} \tau v^{N}(K r) B_{j}^{(N)} e^{r K A^{(N)}} d r
\end{aligned}
$$

where the notation $\overrightarrow{\exp }$ is used to denote the chronological exponent, see [3]. We claim that

$$
\int_{s}^{t} e^{-r K A^{(N)}} v^{N}(K r) B_{j}^{(N)} e^{r K A^{(N)}} d r \rightarrow \frac{t-s}{T}\left(\nu_{0} \mathcal{E}_{0}\left(B_{j}^{(N)}\right)+\nu_{1} \mathcal{E}_{\sigma}\left(B_{j}^{(N)}\right)\right)
$$


as $K \rightarrow \infty$, uniformly with respect to $s, t \in[0, T]$. Indeed, this follows from (9) and the fact that for every $\sigma^{\prime} \in \mathbb{R} \backslash \sigma \mathbb{Z}$

$$
\frac{1}{K} \int_{K s}^{K t} v^{N}(r) e^{i \sigma^{\prime} r} d r \rightarrow 0
$$

as $K \rightarrow \infty$, as it can be seen by developing $v^{N}$ in Fourier series.

Hence by (10), (11), and averaging arguments (see, for instance, [3, Lemma 8.2]) we deduce that

$$
\overrightarrow{\exp } \int_{K s}^{K t} \Theta^{(N)}\left(r, \frac{\tau \omega^{N}(r)}{K}\right) d r \rightarrow \exp \left(\frac{(t-s) \tau}{T}\left(\nu_{0} \mathcal{E}_{0}\left(B_{j}^{(N)}\right)+\nu_{1} \mathcal{E}_{\sigma}\left(B_{j}^{(N)}\right)\right)\right)
$$

as $K \rightarrow \infty$, uniformly with respect to $s, t \in[0, T]$ and $\tau \in[a, b]$.

Step 2. Let

$$
y(t)=e^{-t A} \Upsilon_{t}^{\tau \omega^{N} / K}(\psi),
$$

for $\psi \in \Pi_{n}(\mathcal{H})$. By Lemma 5, $y(t)$ is a solution of $(6)$.

By variation of constants formula and since $\Pi_{N} \Theta(s, u)\left(\mathrm{I}-\Pi_{N}\right)$ is an operator uniformly bounded with respect to $s \in \mathbb{R}$ and $u \in U$, we deduce from (7) that

$$
\begin{aligned}
\Pi_{n} y(t) & =\Pi_{n} \overrightarrow{\exp } \int_{0}^{t} \Theta^{(N)}\left(s, \frac{\tau \omega^{N}(s)}{K}\right) d s(\psi) \\
& +\Pi_{n} \int_{0}^{t}\left(\overrightarrow{\exp } \int_{s}^{t} \Theta^{(N)}\left(r, \frac{\tau \omega^{N}(r)}{K}\right) d r\right) \Pi_{N} \Theta\left(s, \frac{\tau \omega^{N}(s)}{K}\right)\left(\mathrm{I}-\Pi_{N}\right) y(s) d s .
\end{aligned}
$$

We are left to prove that $\lim _{N \rightarrow \infty} \lim _{K \rightarrow \infty} \Pi_{n} e^{-K T A} \Upsilon_{K T}^{\tau \omega^{N}} / K \Pi_{n}=\exp \left(\nu_{0} \mathcal{E}_{0}\left(B_{j}^{(n)}\right)+\nu_{1} \mathcal{E}_{\sigma}\left(B_{j}^{(n)}\right)\right)$ uniformly with respect to $\tau \in[a, b]$. Indeed, by unitarity of the evolution of (6), this also implies that

$$
\lim _{N \rightarrow \infty} \lim _{K \rightarrow \infty}\left(\mathrm{I}-\Pi_{n}\right) e^{-K T A} \Upsilon_{K T}^{\tau \omega^{N} / K} \Pi_{n}=0,
$$

uniformly with respect to $\tau \in[a, b]$.

By (12), for every $N>n$ we have that

$$
\lim _{K \rightarrow \infty} \Pi_{n} \overrightarrow{\exp } \int_{0}^{K T} \Theta^{(N)}\left(s, \frac{\tau \omega^{N}(s)}{K}\right) d s \Pi_{n}=\exp \left(\tau\left(\nu_{0} \mathcal{E}_{0}\left(B_{j}^{(n)}\right)+\nu_{1} \mathcal{E}_{\sigma}\left(B_{j}^{(n)}\right)\right)\right),
$$

uniformly with respect to $\tau \in[a, b]$. Hence, we are left to prove that for every $\varepsilon>0$ and every $N$ large enough one has

$\limsup _{K \rightarrow \infty}\left\|\Pi_{n} \int_{0}^{K T}\left(\overrightarrow{\exp } \int_{s}^{K T} \Theta^{(N)}\left(r, \frac{\tau \omega^{N}(r)}{K}\right) d r\right) \Pi_{N} \Theta\left(s, \frac{\tau \omega^{N}(s)}{K}\right)\left(\mathrm{I}-\Pi_{N}\right) e^{-s A} \Upsilon_{s}^{\tau \omega^{N}} / K d s \Pi_{n}\right\|<\varepsilon$,

uniformly with respect to $\tau \in[a, b]$. Notice that

$$
\begin{aligned}
& \int_{0}^{K T}\left(\overrightarrow{\exp } \int_{s}^{K T} \Theta^{(N)}\left(r, \frac{\tau \omega^{N}(r)}{K}\right) d r\right) \Pi_{N} \Theta\left(s, \frac{\tau \omega^{N}(s)}{K}\right)\left(\mathrm{I}-\Pi_{N}\right) e^{-s A} \Upsilon_{s}^{\tau \omega^{N}} / K d s= \\
& \int_{0}^{T}\left(\overrightarrow{\exp } \int_{K s}^{K T} \Theta^{(N)}\left(r, \frac{\tau \omega^{N}(r)}{K}\right) d r\right) \Pi_{N} \Theta\left(K s, \tau \omega^{N}(K s)\right)\left(\mathrm{I}-\Pi_{N}\right) e^{-K s A} \Upsilon_{K s}^{\tau \omega^{N} / K} d s .
\end{aligned}
$$


We are going to prove, therefore, that for every $\varepsilon>0$ and every $N$ large enough

$$
\limsup _{K \rightarrow \infty}\left\|\Pi_{n}\left(\overrightarrow{\exp } \int_{K s}^{K T} \Theta^{(N)}\left(r, \frac{\tau \omega^{N}(r)}{K}\right) d r\right) \Pi_{N} \Theta\left(K s, \tau \omega^{N}(K s)\right)\left(\mathrm{I}-\Pi_{N}\right)\right\|<\varepsilon,
$$

uniformly with respect to $s \in[0, T]$ and $\tau \in[a, b]$. We have that

$$
\begin{aligned}
\Pi_{n}( & \left.\overrightarrow{\exp } \int_{K s}^{K T} \Theta^{(N)}\left(r, \frac{\tau \omega^{N}(r)}{K}\right) d r\right) \Pi_{N} \Theta\left(K s, \tau \omega^{N}(K s)\right)\left(\mathrm{I}-\Pi_{N}\right) \\
= & \Pi_{n}\left(\overrightarrow{\exp } \int_{K s}^{K T} \Theta^{(N)}\left(r, \frac{\tau \omega^{N}(r)}{K}\right) d r\right) \Pi_{n} \Theta\left(K s, \tau \omega^{N}(K s)\right)\left(\mathrm{I}-\Pi_{N}\right) \\
& +\Pi_{n}\left(\overrightarrow{\exp } \int_{K s}^{K T} \Theta^{(N)}\left(r, \frac{\tau \omega^{N}(r)}{K}\right) d r\right)\left(\Pi_{N}-\Pi_{n}\right) \Theta\left(K s, \tau \omega^{N}(K s)\right)\left(\mathrm{I}-\Pi_{N}\right) .
\end{aligned}
$$

Fix $N$ large enough so that

$$
\left\|\Pi_{n} \Theta(s, u)\left(\mathrm{I}-\Pi_{N}\right)\right\|<\frac{\varepsilon}{T}
$$

for every $s \in \mathbb{R}$ and $u \in U$. Concerning the term in (13), by assumption ( $\mathbb{A} 2$ ) one has that

$$
\left(\Pi_{N}-\Pi_{n}\right) \Theta(s, u)\left(\mathrm{I}-\Pi_{N}\right)
$$

is a bounded operator on $\mathcal{H}$, uniformly with respect to $s \in \mathbb{R}$ and $u \in U$.

Notice that by the assumptions on $\sigma, j, \nu_{0}$, and $\nu_{1}$ it holds

$$
\Pi_{n}\left(\nu_{0} \mathcal{E}_{0}\left(B_{j}^{(N)}\right)+\nu_{1} \mathcal{E}_{\sigma}\left(B_{j}^{(N)}\right)\right)\left(\Pi_{N}-\Pi_{n}\right)=0 .
$$

Hence, by (12),

$$
\Pi_{n}\left(\overrightarrow{\exp } \int_{K s}^{K T} \Theta^{(N)}\left(r, \frac{\tau \omega^{N}(r)}{K}\right) d r\right)\left(\Pi_{N}-\Pi_{n}\right)
$$

tends to 0 as $K \rightarrow \infty$ uniformly with respect to $s \in[0, T]$ and $\tau \in[a, b]$. Hence the term in (13) goes to 0 as $K \rightarrow \infty$ uniformly with respect to $\tau \in[a, b]$.

\subsection{Efficiency of admissible controls}

Let us discuss the values of $\nu_{0}$ and $\nu_{1}$ which can be obtained with a control satisfying the hypothesis of Lemma 6. We distinguish two cases depending on the nature of the control set $U_{j}$.

If $U_{j}=[-\delta, \delta]$ then for every $\nu_{0}, \nu_{1} \in \mathbb{R}$ a simple example of periodic function, independent of $N$, satisfying (8) and (9) is given by

$$
v(t)=\frac{\sigma}{2 \pi}\left(\nu_{0}+2 \nu_{1} \cos (\sigma t)\right) .
$$

Indeed by orthogonality of trigonometric functions one has that

$$
\int_{0}^{T} v(t) e^{i m \sigma t} d t=0
$$

for every integer $m \geq 2$ (in particular for every $m \geq 2$ such that $m \sigma \in \Sigma_{N}$ ). 
In the case in which $U_{j}=[0, \delta]$ the restriction on the sign of $v^{N}$ represents an additional requirement. If $\nu_{0}=0$ then $v^{N}=0$. Otherwise, up to replacing $v^{N}$ by $v^{N} / \nu_{0}$, one can assume that $\nu_{0}=1$. In this case, following [13, Section 2.4] we call $\left|\nu_{1}\right|$ the efficiency of the control $v^{N}$. Notice, for instance, that the efficiency of the functions $\frac{\sigma}{2 \pi}(1 \pm \cos (\sigma t))$ is $1 / 2$. Hence, by convexity, one can choose a $v^{N}$, independent of $N$, so that $\nu_{0}=1$ and $\nu_{1}$ is any prescribed value in $[-1 / 2,1 / 2]$.

However, in both cases, these simple and explicit choices of $v^{N}$ are somehow too rigid for our purposes. Indeed these functions are not piecewise constant and, on the other hand, one cannot concatenate smooth functions of the form (14) corresponding to distinct values of $\sigma$ in a smooth (i.e. $C^{\infty}$ ) way. In order to overcome this issue we restrict condition (9) to $m \geq 2$ such that $m \sigma \in \Sigma_{N}$ allowing $v^{N}$ to depend on $N$. This is the rationale for Lemma 7 below, which shows that small perturbations of functions of the form (14) yield admissible controls with efficiency arbitrarily close to $1 / 2$. In the $C^{\infty}$ case we further prescribe the approximating admissible controls to be zero with all derivatives at 0 and $T$ so that concatenations of function of this kind are smooth.

Lemma 7. Let $\sigma>0$ and define $T=2 \pi / \sigma$. Denote by $\mathscr{U}_{T}$ either the class of nonnegative piecewise constant functions on $[0, T]$ or the class of nonnegative $C^{\infty}$ functions on $[0, T]$ that are zero with all derivatives at 0 and $T$. Then, for every $\nu_{1} \in(-1 / 2,1 / 2)$ and $N \in \mathbb{N}$ there exists $w \in \mathscr{U}_{T}$ such that

(i) $\int_{0}^{T} w(t) d t=1$,

(ii) $\int_{0}^{T} w(t) e^{i \sigma t} d t=\nu_{1}$

(iii) $\int_{0}^{T} w(t) e^{i m \sigma t} d t=0$ for every $m \geq 2$ such that $m \sigma \in \Sigma_{N}$.

Proof. Let $\varphi_{0} \in\{t \mapsto 1-\cos (\sigma t), t \mapsto 1+\cos (\sigma t)\}$ and

$$
\left\{\varphi_{1}, \ldots, \varphi_{k}\right\}=\left\{t \mapsto \sin (m \sigma t), t \mapsto \cos (m \sigma t) \mid m \geq 2 \text { such that } m \sigma \in \Sigma_{N}\right\} .
$$

Let $\alpha \in[4 / 5,1)$. For every $\varepsilon>0$ consider $w_{0}, w_{1}, \ldots, w_{k} \in \mathscr{U}_{T}$ such that

$$
\begin{gathered}
w_{0}(t) \geq \varepsilon^{\alpha} \quad \text { for every } t \in[\varepsilon, T-\varepsilon], \\
w_{0}(t)=0 \quad \text { for every } t \in\left[0, \varepsilon^{2}\right) \cup\left(T-\varepsilon^{2}, T\right] \\
w_{j}(t)=0 \quad \text { for every } j=1, \ldots, k, \text { and } t \in[0, \varepsilon) \cup(T-\varepsilon, T],
\end{gathered}
$$

and such that

$$
\left\|w_{j}-\varphi_{j}\right\|_{L^{2}([0, T])}<C \varepsilon, \quad \text { for every } j=0, \ldots, k,
$$

for some $C$ independent of $\varepsilon$. Indeed, since $\alpha \geq 4 / 5$ one easily checks that (15) is compatible with (16).

Consider the solution $\left(c_{1}, \ldots, c_{k}\right)$ of the linear system

$$
\left\{\begin{array}{cc}
\left\langle w_{1}, \varphi_{1}\right\rangle c_{1}+\cdots+\left\langle w_{k}, \varphi_{1}\right\rangle c_{k} & =-\left\langle w_{0}, \varphi_{1}\right\rangle \\
\vdots & \vdots \\
\left\langle w_{1}, \varphi_{k}\right\rangle c_{1}+\cdots+\left\langle w_{k}, \varphi_{k}\right\rangle c_{k} & =-\left\langle w_{0}, \varphi_{k}\right\rangle
\end{array}\right.
$$

Notice that the solution exists since the matrix of the system is close to an invertible diagonal matrix for $\varepsilon$ sufficiently small. Moreover $\left|c_{j}\right| \leq C \varepsilon$ for every $j=1, \ldots, k$ (possibly considering a larger constant $C$ ). 
Then define

$$
w=\frac{w_{0}}{T}+\sum_{j=1}^{k} c_{j} w_{j} .
$$

The function $w$ belongs to $\mathscr{U}_{T}$. Indeed since $\alpha<1$ then $w \geq 0$ for $\varepsilon$ small enough.

By construction $w$ satisfies (iii). Possibly rescaling $w$ by a factor $\left(\int_{0}^{T} w d t\right)^{-1}=1+O(\varepsilon)$ point (i) is satisfied. Notice now that

$$
\int_{0}^{T} w(t) e^{i \sigma t} d t=\frac{1}{T} \int_{0}^{T} \varphi_{0}(t) e^{i \sigma t} d t+O(\varepsilon)= \pm \frac{1}{2}+O(\varepsilon)
$$

The conclusion follows by convexity and letting $\varepsilon$ go to zero.

Remark 5 . One could relax the condition on $v^{N}$ in (9) by replacing the set $\Sigma_{N}$ by

$$
\left\{\left|\lambda_{l}-\lambda_{k}\right| \mid l, k=1, \ldots, N, \text { and }\left\langle\phi_{l}, B_{j} \phi_{k}\right\rangle \neq 0\right\}
$$

The proof of Lemma 6 remains unchanged since the condition in (9) is only used in (11).

\section{Proof of Theorem 2}

\subsection{Finite-dimensional exact controllability implies infinite-dimensional approxi- mate controllability up to phases}

Let $n_{0} \in \mathbb{N}$ and $n>n_{0}$ be given by the Lie-Galerkin Control Condition. Define the collection of matrices

$$
\begin{aligned}
\mathcal{W}_{n}= & \left\{A^{(n)}\right\} \cup\left\{\mathcal{E}_{0}\left(B_{j}^{(n)}\right) \mid(0, j) \in \Xi_{n}\right\} \\
& \cup\left\{\mathcal{E}_{0}\left(B_{j}^{(n)}\right)+\nu \mathcal{E}_{\sigma}\left(B_{j}^{(n)}\right) \mid(\sigma, j) \in \Xi_{n} \text { and } \sigma, j \text { are such that }(0, j) \in \Xi_{n}, \sigma \neq 0, \nu \in(-1 / 2,1 / 2)\right\} \\
& \cup\left\{\mathcal{E}_{\sigma}\left(B_{j}^{(n)}\right) \mid(\sigma, j) \in \Xi_{n}, \sigma \neq 0, \text { and } U_{j}=[-\delta, \delta]\right\},
\end{aligned}
$$

where $\Xi_{n}$ is defined as in (4). The matrices in $\mathcal{W}_{n}$ correspond to the asymptotic dynamics obtained in Lemma 6 for the admissible choices of values for $\nu_{0}$ and $\nu_{1}$ (see Lemma 7 ). Notice that $\operatorname{Lie}\left(\mathcal{W}_{n}\right)=$ $\operatorname{Lie}\left(\mathcal{M}_{n}\right)$ and, by the Lie-Galerkin Control Condition, $\operatorname{Lie}\left(\mathcal{W}_{n}\right) \supseteq \mathfrak{s u}(n)$.

Consider the auxiliary control system

$$
\dot{x}=M(t) x, \quad M(t) \in \mathcal{W}_{n},
$$

where $M$ plays the role of control.

Proposition 8 below, based on Lemma 6, states that any propagator of (17) can be approximated by a propagator of system (2).

Proposition 8. Let $n, k \in \mathbb{N}, a, b \in \mathbb{R}$ with $0<a<b$, and $M_{1}, \ldots, M_{k} \in \mathcal{W}_{n}$. For every $\varepsilon>0$ and $\tau_{1}, \ldots, \tau_{k} \in[a, b]$ there exist $u \in \mathcal{U}, T_{u}>0$, and $\gamma \geq 0$ such that

$$
\left\|\Upsilon_{T_{u}}^{u}-e^{\gamma A} \circ e^{\tau_{k} M_{k}} \circ \cdots \circ e^{\tau_{1} M_{1}}\right\|_{L\left(\Pi_{n}(\mathcal{H}), \mathcal{H}\right)}<\varepsilon
$$


where every $e^{\tau_{\ell} M_{\ell}}$ is identified with an operator on $\mathcal{H}$. Moreover, $\gamma$ can be taken independent of $\tau_{1}, \ldots, \tau_{k} \in[a, b]$.

More precisely, for $\ell=1, \ldots, k$ and $\tau_{1}, \ldots, \tau_{k} \in[a, b]$ there exist $T_{\ell} \geq 0$ and $\omega_{\ell}:\left[0, T_{\ell}\right] \rightarrow \mathbb{R}^{p}$, such that for $K$ large enough $u$ can be taken as the concatenation

$$
u=\left.\left.\left.0\right|_{\left[0, \chi_{1}\right]} * \underbrace{\frac{\tau_{1} \omega_{1}}{K} * \cdots * \frac{\tau_{1} \omega_{1}}{K}}_{K \text {-times }} * 0\right|_{\left[0, \chi_{2}\right]} * \underbrace{\frac{\tau_{2} \omega_{2}}{K} * \cdots * \frac{\tau_{2} \omega_{2}}{K}}_{K \text {-times }} * \cdots * 0\right|_{\left[0, \chi_{k}\right]} * \underbrace{\frac{\tau_{k} \omega_{k}}{K} * \cdots * \frac{\tau_{k} \omega_{k}}{K}}_{K-\text { times }}
$$

with $\chi_{\ell} \geq 0$ continuously depending on $\tau_{\ell}$, where $\left.0\right|_{\left[0, \chi_{\ell}\right]}$ denotes the function $\left[0, \chi_{\ell}\right] \ni t \mapsto(0, \ldots, 0) \in$ $\mathbb{R}^{p}$.

Proof. We construct $u$ as the concatenation $u^{1} * \cdots * u^{k}$ of $k$ controls $u^{\ell}:\left[0, \vartheta_{\ell}\right] \rightarrow \mathbb{R}^{p}, \ell=1, \ldots, k$, namely, $u(t)=u^{\ell}\left(t-\left(\vartheta_{1}+\cdots+\vartheta_{\ell-1}\right)\right)$ for $t \in\left[\vartheta_{1}+\cdots+\vartheta_{\ell-1}, \vartheta_{1}+\cdots+\vartheta_{\ell}\right)$, with $u^{\ell}=\left.0\right|_{\left[0, \chi_{\ell}\right]} * \frac{\tau_{\ell} \omega_{\ell}}{K} *$ $\cdots * \frac{\tau_{\ell} \omega_{\ell}}{K}$ and $\vartheta_{\ell}=\chi_{\ell}+K T_{\ell}$.

If $\mathcal{U}$ is the set of piecewise constant functions with values in $U$ then this concatenation clearly results in an admissible control. If $\mathcal{U}$ is the set of $C^{\infty}$ functions with values in $U$ then, in order to guarantee that $u$ is admissible, every control $u^{\ell}$ is constructed as a smooth function which is zero with all derivatives at 0 and $\vartheta_{\ell}$.

Let us construct recursively $u^{\ell}$ and $\vartheta_{\ell}$ for $\ell \in\{1, \ldots, k\}$.

- For $\ell=1$ we have two cases.

- If $M_{1}=A^{(n)}$ then consider $T_{1}=0$ and $\chi_{1}=\tau_{1}$.

- If $M_{1}$ is of the form $\nu_{0} \mathcal{E}_{0}\left(B_{j}^{(n)}\right)+\nu_{1} \mathcal{E}_{\sigma}\left(B_{j}^{(n)}\right)$ for some $\sigma>0$ and $j \in\{1, \ldots, p\}$ then we apply Lemma 6 . Therefore there exist $N>n$, a control $\omega^{N}$ and $K$ such that

$$
\left\|\Upsilon_{K T}^{\tau_{1} \omega^{N} / K}-e^{K T A} e^{\tau_{1} M_{1}}\right\|_{L\left(\Pi_{n}(\mathcal{H}), \mathcal{H}\right)}<\varepsilon / k
$$

for every $\tau_{1} \in[a, b]$, where $T=2 \pi / \sigma$. Then, $T_{1}=T, \chi_{1}=0, \omega_{1}=\left.\omega^{N}\right|_{[0, T]}$.

- Let $\ell \geq 2$ and assume that, for every $\tau_{1}, \ldots, \tau_{\ell-1} \in[a, b]$, the control $u$ is constructed on $\left[0, \vartheta_{1}+\cdots+\vartheta_{\ell}\right)$. Then there exists $\gamma \geq 0$ such that

$$
\left\|e^{-\gamma A} \Upsilon_{\vartheta_{1}+\cdots+\vartheta_{\ell}}^{u}-e^{\tau_{\ell-1} M_{\ell-1}} \circ \cdots \circ e^{\tau_{1} M_{1}}\right\|_{L\left(\Pi_{n}(\mathcal{H}), \mathcal{H}\right)}<\varepsilon \frac{\ell-1}{k},
$$

for every $\tau_{1}, \ldots, \tau_{\ell-1} \in[a, b]$. Again we distinguish two cases.

- If $M_{\ell}=A^{(n)}$ then set $T_{\ell}=0$ and $\chi_{\ell}=\tau_{\ell}$.

- Consider now the case in which $M_{\ell}$ is of the form $\nu_{0} \mathcal{E}_{0}\left(B_{j}^{(n)}\right)+\nu_{1} \mathcal{E}_{\sigma}\left(B_{j}^{(n)}\right)$ for some $\sigma>0$ and $j \in\{1, \ldots, p\}$.

Take a time $\gamma^{\prime} \geq 0$ such that $\gamma^{\prime}+\gamma$ is an integer multiple of $T=2 \pi / \sigma$, for instance $\gamma^{\prime}=\left\lceil\frac{\gamma}{T}\right\rceil T-\gamma$, and let $\chi_{\ell}=\gamma^{\prime}$. Apply now Lemma 6. Then there exist $N>n$ a control $\omega^{N}$ and $K$ such that

$$
\left\|\Upsilon_{K T}^{\tau_{\ell} \omega^{N} / K}-e^{K T A} e^{\tau_{\ell} M_{\ell}}\right\|_{L\left(\Pi_{n}(\mathcal{H}), \mathcal{H}\right)}<\varepsilon / k,
$$

for every $\tau_{\ell} \in[a, b]$. 
Since $\gamma^{\prime}+\gamma$ is an integer multiple of $T=2 \pi / \sigma$, we have that $\left[M_{\ell}, e^{-\left(\gamma+\gamma^{\prime}\right) A}\right]=0$, which implies that $\left[e^{\tau_{\ell} M_{\ell}}, e^{-\left(\gamma+\gamma^{\prime}\right) A}\right]=0$. Hence

$$
\begin{aligned}
& \left\|e^{-\left(\gamma+\gamma^{\prime}+K T\right) A} \Upsilon_{\vartheta_{1}+\cdots+\vartheta_{\ell+1}}^{u}-e^{\tau_{\ell} M_{\ell}} \circ \cdots \circ e^{\tau_{1} M_{1}}\right\|_{L\left(\Pi_{n}(\mathcal{H}), \mathcal{H}\right)} \\
& =\left\|e^{-\left(\gamma+\gamma^{\prime}+K T\right) A} \Upsilon_{K T}^{\tau_{\ell} \omega^{N} / K} e^{\gamma^{\prime} A} \Upsilon_{\vartheta_{1}+\cdots+\vartheta_{\ell}}^{u}-e^{\tau_{\ell} M_{\ell}} \circ \cdots \circ e^{\tau_{1} M_{1}}\right\|_{L\left(\Pi_{n}(\mathcal{H}), \mathcal{H}\right)} \\
& \leq\left\|\Upsilon_{K T}^{\tau_{\ell} \omega^{N} / K}-e^{K T A} e^{\tau_{\ell} M_{\ell}}\right\|_{L\left(\Pi_{n}(\mathcal{H}), \mathcal{H}\right)} \\
& +\left\|e^{-\left(\gamma+\gamma^{\prime}\right) A} e^{\tau_{\ell} M_{\ell}} \Upsilon_{\vartheta_{1}+\cdots+\vartheta_{\ell}}^{u}-e^{\tau_{\ell} M_{\ell}} \circ \cdots \circ e^{\tau_{1} M_{1}}\right\|_{L\left(\Pi_{n}(\mathcal{H}), \mathcal{H}\right)} \\
& \leq \frac{\varepsilon}{k}+\left\|e^{-\gamma A} \Upsilon_{\vartheta_{1}+\cdots+\vartheta_{\ell}}^{u}-e^{\tau_{\ell-1} M_{\ell-1}} \circ \cdots \circ e^{\tau_{1} M_{1}}\right\|_{L\left(\Pi_{n}(\mathcal{H}), \mathcal{H}\right)} \leq \frac{\varepsilon \ell}{k},
\end{aligned}
$$

which concludes the induction with $T_{\ell}=T$ and $\omega_{\ell}=\left.\omega^{N}\right|_{[0, T]}$ and with the role of $\gamma$ in (18) now played by $\gamma+\gamma^{\prime}+K T$.

This concludes the construction of $u$ with $T_{u}=\vartheta_{1}+\cdots+\vartheta_{k}$.

Finally, notice that, in the case in which $\mathcal{U}$ is the set of $C^{\infty}$ functions with values in $U$, thanks to Lemma 7 at each step we can assume that the control $\omega_{\ell}$ is zero with all derivatives at 0 and $T$ (and so the control $u$ is admissible). Indeed, if $\nu_{0} \neq 0$ then Lemma 7 applies directly, while if $\nu_{0}=0$ then $\omega_{\ell}$ can be defined as the linear combination of two nonnegative controls associated with two distinct values of $\nu_{1}$ in Lemma 7 .

Remark 6. Lemma 7 and Proposition 8 are the only steps in the proof of Theorems 3 and 2 where the nature of the class $\mathcal{U}$ is used. Actually, the proofs of both results are still valid under the following assumptions on the class $\mathcal{U}$ : there exists a subclass $\mathcal{V}$ of $\mathcal{U}$ which is convex and closed under concatenation, which contains the null function, and such that Lemma 7 holds with $\cup_{T>0} \mathscr{U}_{T}=\mathcal{V}$.

\subsection{Phase tuning}

The controllability of (17), ensured by the Lie-Galerkin Control Condition, together with Proposition 8 implies approximate controllability up to phases of system (2). In this section we show how to correct the dephasing term $e^{\gamma A}$ appearing in Proposition 8 by letting the system evolve freely for a suitable amount $\tau$ of time, as proved below (see Figure 1).

Lemma 9. Let $\psi \in \mathcal{H}, \mu>0, N \in \mathbb{N}$, and $\mathcal{N}$ be a neighborhood of $\Pi_{N}\left(e^{\mu A} \psi\right)$ in $\operatorname{span}\left\{\phi_{1}, \ldots, \phi_{N}\right\}$. Then there exists $\tau \geq 0$ such that $e^{\tau A} \mathcal{N}$ is a neighborhood of $\Pi_{N} \psi$ in $\operatorname{span}\left\{\phi_{1}, \ldots, \phi_{N}\right\}$.

Proof. Without loss of generality we can assume that $\mathcal{N}$ is an open ball $B_{2 \varepsilon}\left(\Pi_{N}\left(e^{\mu A} \psi\right)\right)$ of radius $2 \varepsilon$ centered at $\Pi_{N}\left(e^{\mu A} \psi\right)$, for some $\varepsilon>0$.

Now, consider the sequence $\left(\beta_{k}\right)_{k \in \mathbb{N}}$ of sets

$$
\beta_{k}=B_{\varepsilon}\left(\Pi_{N}\left(e^{k \mu A} \psi\right)\right)
$$

All the elements of the sequence $\left(\beta_{k}\right)_{k \in \mathbb{N}}$ are of constant positive volume and are contained in the common subset $B_{\left\|\Pi_{N}(\psi)\right\|+\varepsilon}(0)$ of $\operatorname{span}\left\{\phi_{1}, \ldots, \phi_{N}\right\}$. Since the latter has finite volume, there exist two integers $\ell, m \geq 1$ such that $\beta_{\ell} \cap \beta_{\ell+m} \neq \emptyset$. Since $e^{-\ell \mu A}$ is an isomorphism of $\operatorname{span}\left\{\phi_{1}, \ldots, \phi_{N}\right\}$ we deduce that

$$
B_{\varepsilon}\left(\Pi_{N}(\psi)\right) \cap B_{\varepsilon}\left(\Pi_{N}\left(e^{m \mu A} \psi\right)\right) \neq \emptyset .
$$




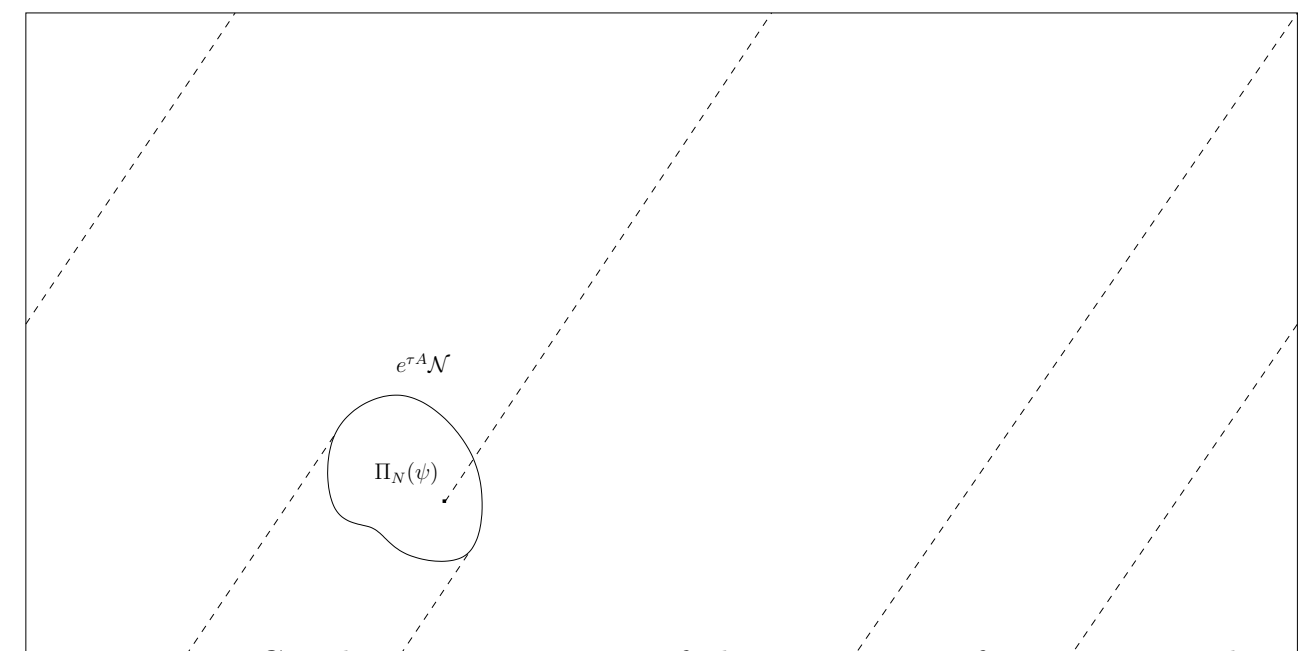

Figure' 1: Graphic'representation of the statément of Lemíma 9. The main idea underlying phase tuning is that the dephasing between solutionis of (2) and,(6) is along $A$. Here $N=2$ and $\Pi_{2}\left(e^{t A} \psi\right)=$ $\left(e^{i t \lambda_{1}}\left\langle\phi_{1}, \psi\right\rangle, e^{i t \lambda_{2}^{\prime}}\left\langle\phi_{2}, \psi\right\rangle\right)$ is identified, using the phases, of its components, with elements of the torus $\mathbb{S}^{1} \times \mathbb{S}^{1}$.
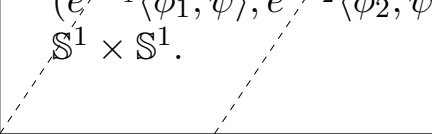

Therefore

$$
\Pi_{N}(\psi) \in B_{2 \varepsilon}\left(\Pi_{N}\left(e^{m \mu A} \psi\right)\right)=e^{(m-1) \mu A} B_{2 \varepsilon}\left(\Pi_{N}\left(e^{\mu A} \psi\right)\right)=e^{(m-1) \mu A} \mathcal{N},
$$

which proves the lemma with $\tau=(m-1) \mu$.

\subsection{Final step}

Proof of Theorem 2. Let $\psi_{0}$ and $\psi_{1}$ be in the unit sphere of $\mathcal{H}$. For $\varepsilon>0$ let $n_{0} \in \mathbb{N}$ be such that

$$
\left\|\psi_{0}-\frac{\Pi_{n_{0}} \psi_{0}}{\left\|\Pi_{n_{0}} \psi_{0}\right\|}\right\|<\frac{\varepsilon}{3} \quad \text { and } \quad\left\|\psi_{1}-\frac{\Pi_{n_{0}} \psi_{1}}{\left\|\Pi_{n_{0}} \psi_{1}\right\|}\right\|<\frac{\varepsilon}{3} .
$$

The Lie-Galerkin condition ensures the existence of $n>n_{0}$ such that system (17) is controllable. By Proposition 8 there exist $u \in \mathcal{U}, T_{u} \geq 0$, and $\gamma \geq 0$ such that

$$
\left\|\Upsilon_{T_{u}}^{u}\left(\frac{\Pi_{n_{0}} \psi_{0}}{\left\|\Pi_{n_{0}} \psi_{0}\right\|}\right)-e^{\gamma A} \frac{\Pi_{n_{0}} \psi_{1}}{\left\|\Pi_{n_{0}} \psi_{1}\right\|}\right\|<\frac{\varepsilon}{3} .
$$


By triangular inequality we have that

$$
\begin{aligned}
& \left\|\Upsilon_{T_{u}}^{u}\left(\psi_{0}\right)-e^{\gamma A} \psi_{1}\right\| \leq\left\|\Upsilon_{T_{u}}^{u}\left(\psi_{0}\right)-\Upsilon_{T_{u}}^{u}\left(\frac{\Pi_{n_{0}} \psi_{0}}{\left\|\Pi_{n_{0}} \psi_{0}\right\|}\right)\right\| \\
& +\left\|\Upsilon_{T_{u}}^{u}\left(\frac{\Pi_{n_{0}} \psi_{0}}{\left\|\Pi_{n_{0}} \psi_{0}\right\|}\right)-e^{\gamma A} \frac{\Pi_{n_{0}} \psi_{1}}{\left\|\Pi_{n_{0}} \psi_{1}\right\|}\right\|+\left\|e^{\gamma A} \frac{\Pi_{n_{0}} \psi_{1}}{\left\|\Pi_{n_{0}} \psi_{1}\right\|}-e^{\gamma A} \psi_{1}\right\| \\
& =\left\|\psi_{0}-\frac{\Pi_{n_{0}} \psi_{0}}{\left\|\Pi_{n_{0}} \psi_{0}\right\|}\right\| \\
& +\left\|\Upsilon_{T_{u}}^{u}\left(\frac{\Pi_{n_{0}} \psi_{0}}{\left\|\Pi_{n_{0}} \psi_{0}\right\|}\right)-e^{\gamma A} \frac{\Pi_{n_{0}} \psi_{1}}{\left\|\Pi_{n_{0}} \psi_{1}\right\|}\right\|+\left\|\frac{\Pi_{n_{0}} \psi_{1}}{\left\|\Pi_{n_{0}} \psi_{1}\right\|}-\psi_{1}\right\|<\varepsilon .
\end{aligned}
$$

Applying Lemma 9 there exists a time $\tau \geq 0$ such that $e^{\tau A} \Upsilon_{T_{u}}^{u}\left(\psi_{0}\right)$ is $\varepsilon$-close to $\psi_{1}$. The concatenation of $u$ and the zero function for a time $\tau$ then steers $\psi_{0}$ into a $\varepsilon$-neighborhood of $\psi_{1}$. Notice that such a concatenation is admissible since, in the case in which $\mathcal{U}$ is the set of $C^{\infty}$ functions with values in $U$, the control $u$, given by Proposition 8 , is zero with all derivatives at $T_{u}$.

\section{$5 \quad$ Proof of Theorem 3}

Theorem 3 states that under the Lie-Galerkin Control Condition it is possible to control approximately system (2) and, at the same time, to control it exactly in projection on a prescribed number of components. The first step in the proof consists in showing that the approximate controllability part is a consequence of the exact controllability in projections.

\subsection{Exact controllability in projections implies approximate controllability}

Lemma 10. Assume that for every $n_{0} \in \mathbb{N}, \psi_{1} \in \mathcal{H}$ with $\left\|\psi_{1}\right\|=1$, and $\psi_{2} \in \mathcal{H}$ such that $\left\|\psi_{2}\right\|=1$ and $\left\|\Pi_{n_{0}}\left(\psi_{2}\right)\right\|<1$, there exist $u \in \mathcal{U}$ and $T>0$ such that

$$
\Pi_{n_{0}}\left(\Upsilon_{T}^{u}\left(\psi_{1}\right)\right)=\Pi_{n_{0}}\left(\psi_{2}\right)
$$

Then for every $\mathfrak{n} \in \mathbb{N}, \psi_{\text {in }} \in \mathcal{H}$ with $\left\|\psi_{\text {in }}\right\|=1$, and $\psi_{\mathrm{f}} \in \mathcal{H}$ such that $\left\|\psi_{\mathrm{f}}\right\|=1$ and $\left\|\Pi_{\mathfrak{n}}\left(\psi_{\mathrm{f}}\right)\right\|<1$, and for every $\varepsilon>0$, there exist $u \in \mathcal{U}$ and $T>0$ such that

$$
\Pi_{\mathfrak{n}}\left(\Upsilon_{T}^{u}\left(\psi_{\mathrm{in}}\right)\right)=\Pi_{\mathfrak{n}}\left(\psi_{\mathrm{f}}\right) \quad \text { and } \quad\left\|\Upsilon_{T}^{u}\left(\psi_{\mathrm{in}}\right)-\psi_{\mathrm{f}}\right\|<\varepsilon
$$

Proof. Let $\mathfrak{n}, \varepsilon, \psi_{\mathrm{in}}$, and $\psi_{\mathrm{f}}$ as above. If $\psi_{\mathrm{f}} \in \mathcal{H}$ has an infinite number of non-zero components then it is sufficient to take $n_{0} \geq \mathfrak{n}$ such that $\left\|\Pi_{n_{0}} \psi_{\mathrm{f}}-\psi_{\mathrm{f}}\right\|<\varepsilon / 2$ and apply (19) with $\psi_{1}=\psi_{\text {in }}$ and $\psi_{2}=\psi_{\mathrm{f}}$.

If, instead, there exists $n_{0} \in \mathbb{N}$ such that $\left\langle\phi_{n_{0}}, \psi_{\mathrm{f}}\right\rangle \neq 0$ and $\left\langle\phi_{k}, \psi_{\mathrm{f}}\right\rangle=0$ for every $k>n_{0}$ then note that $n_{0}>\mathfrak{n}$ and consider $\psi_{2}$ defined component-wise as

$$
\left\langle\phi_{k}, \psi_{2}\right\rangle= \begin{cases}\left\langle\phi_{k}, \psi_{\mathrm{f}}\right\rangle & \text { if } k=1, \ldots, n_{0}-1, \\ \sqrt{1-\eta^{2}}\left\langle\phi_{n_{0}}, \psi_{\mathrm{f}}\right\rangle & \text { if } k=n_{0}, \\ \eta\left\langle\phi_{n_{0}}, \psi_{\mathrm{f}}\right\rangle & \text { if } k=n_{0}+1, \\ 0 & \text { if } k>n_{0}+1,\end{cases}
$$

for $\eta<\varepsilon / 2$. Then apply (19) with $\psi_{1}=\psi_{\text {in }}$. 


\section{$5.2 \quad$ A useful topological tool}

The following topological result is standard in degree theory. We provide its proof for completeness. Similar results used in the control literature are, for instance, [2, Lemma 7] and [18, Lemma 4.1].

Lemma 11. Let $X \subset \mathbb{R}^{n}$ be open and bounded and let $F \in C\left(\bar{X}, \mathbb{R}^{n}\right)$ be a homeomorphism between $X$ and $F(X)$. Assume that $y_{0}$ is in $F(X)$ and consider $0<\varepsilon \leq \operatorname{dist}\left(y_{0}, F(\partial X)\right)$. If $G \in C\left(\bar{X}, \mathbb{R}^{n}\right)$ satisfies

$$
\max _{x \in \partial X}|F(x)-G(x)|<\varepsilon
$$

then $y_{0} \in \operatorname{int}(G(X))$.

Proof. Consider the homotopy $h \in C\left([0,1] \times \bar{X}, \mathbb{R}^{n}\right)$ defined by $h(t, x)=t F(x)+(1-t) G(x)$. By definition of $\varepsilon$ we have that $h(t, x) \neq y_{0}$ for every $t \in[0,1]$ and $x \in \partial X$. In particular the topological degree $\mathrm{d}\left(h(t, \cdot), X, y_{0}\right)$ is well defined for every $t \in[0,1]$. Since $\left.F\right|_{X}$ is a homeomorphism and $y_{0} \in F(X)$ then

$$
\mathrm{d}\left(F, X, y_{0}\right) \neq 0
$$

and by homotopy invariance

$$
\mathrm{d}\left(G, X, y_{0}\right) \neq 0
$$

which implies that $y_{0} \in \operatorname{int}(G(X))$.

\subsection{Normal controllability}

In this section we consider $\psi_{1}$ and $\psi_{2}$ unit vectors in $\mathcal{H}$ and $n_{0} \in \mathbb{N}$ such that $\left\|\Pi_{n_{0}}\left(\psi_{2}\right)\right\|<1$. Let $n>n_{0}$ be such that the Lie-Galerkin Control Condition holds. Let now $\tilde{\psi}_{2} \in S^{2 n-1}$ be such that $\Pi_{n_{0}}\left(\psi_{2}\right)=\Pi_{n_{0}}\left(\tilde{\psi}_{2}\right)$. By classical results of controllability (see [21] and [34, Theorem 4.3]) system (17) is normally controllable at $\tilde{\psi}_{2}$, in the sense that there exist $M_{1}, \ldots, M_{k} \in \mathcal{W}_{n}$ and $t_{1}, \ldots, t_{k}>0$ such that the map

$$
E:\left(s_{1}, \ldots, s_{k}\right) \mapsto e^{s_{k} M_{k}} \circ \cdots \circ e^{s_{1} M_{1}}\left(\phi_{1}\right)
$$

has rank $2 n-1$ at $\left(t_{1}, \ldots, t_{k}\right)$ and

$$
E\left(t_{1}, \ldots, t_{k}\right)=\tilde{\psi}_{2} .
$$

Since $n>n_{0}$ and $\left\|\Pi_{n_{0}}\left(\tilde{\psi}_{2}\right)\right\|<1$, there exist $j_{1}, \ldots, j_{2 n_{0}} \in\{1, \ldots, k\}$ such that the map

$$
F\left(s_{j_{1}}, \ldots, s_{j_{2 n_{0}}}\right) \mapsto \Pi_{n_{0}}\left(E\left(t_{1}, \ldots, t_{j_{1}-1}, s_{j_{1}}, t_{j_{1}+1}, \ldots, t_{j_{2 n_{0}}-1}, s_{j_{2 n_{0}}}, t_{j_{2 n_{0}}+1}, \ldots, t_{k}\right)\right)
$$

has rank $2 n_{0}$ at $\left(t_{j_{1}}, \ldots, t_{j_{2 n_{0}}}\right)$ and

$$
F\left(t_{j_{1}}, \ldots, t_{j_{2 n_{0}}}\right)=\Pi_{n_{0}}\left(\psi_{2}\right) \quad\left(=\Pi_{n_{0}}\left(\tilde{\psi}_{2}\right)\right) .
$$

Now let $\rho>0$ be such that

$$
X:=B_{\rho}\left(t_{j_{1}}, \ldots, t_{j_{2 n_{0}}}\right) \subset(0,+\infty)^{2 n_{0}}
$$

and $F$ is a diffeomorphism between $X$ and $F(X)$. Let

$$
\eta=\inf _{\left(s_{1}, \ldots, s_{2 n_{0}}\right) \in \partial X}\left\|F\left(s_{1}, \ldots, s_{2 n_{0}}\right)-\Pi_{n_{0}}\left(\psi_{2}\right)\right\|,
$$

and note that $\eta>0$. 
Lemma 12. There exist $\gamma \geq 0$ and a map associating with every $\left(s_{1}, \ldots, s_{2 n_{0}}\right) \in \bar{X}$ a control $v^{s} \in \mathcal{U}$ and $T_{s}>0$ such that the image of the mapping

$$
G: \begin{array}{ccc}
\bar{X} & \rightarrow & \operatorname{span}\left\{\phi_{1}, \ldots, \phi_{n_{0}}\right\} \\
\left(s_{1}, \ldots, s_{2 n_{0}}\right) & \mapsto & \Pi_{n_{0}}\left(\Upsilon_{T_{s}}^{v^{s}}\left(\psi_{1}\right)\right)
\end{array}
$$

contains $\Pi_{n_{0}}\left(e^{\gamma A} \psi_{2}\right)$ in its interior.

Proof. Let $\eta$ be as in (20). By Theorem 2 there exists $w \in \mathcal{U}$ steering $\psi_{1}, \eta / 2$-close to $\phi_{1}$.

Applying Proposition 8 with

$$
\left(\tau_{1}, \ldots, \tau_{k}\right)=\left(t_{1}, \ldots, t_{j_{1}-1}, s_{j_{1}}, t_{j_{1}+1}, \ldots, t_{j_{2 n_{0}}-1}, s_{j_{2 n_{0}}}, t_{j_{2 n_{0}}+1}, \ldots, t_{k}\right),
$$

we deduce the existence of a family of controls $u^{s}$, depending continuously on $s \in X$ such that

$$
\max _{s \in \bar{X}}\left\|F(s)-e^{-\gamma A} \Pi_{n_{0}}\left(\Upsilon_{T_{u}^{s}}^{u^{s}} \phi_{1}\right)\right\|<\eta / 2
$$

Define $v^{s}$ as the concatenation of $w$ and $u^{s}$. The continuity of $G$ with respect to $s$ is ensured by Assumption (A5). The conclusion then follows from Lemma 11.

\subsection{Final step}

We are now ready to conclude the proof of Theorem 3.

Proof of Theorem 3. By Lemma 10 it enough to prove that for every $n_{0} \in \mathbb{N}, \psi_{1} \in \mathcal{H}$ with $\left\|\psi_{1}\right\|=1$, and $\psi_{2} \in \mathcal{H}$ such that $\left\|\psi_{2}\right\|=1$ and $\left\|\Pi_{n_{0}}\left(\psi_{2}\right)\right\|<1$, there exist $u \in \mathcal{U}$ and $T>0$ such that

$$
\Pi_{n_{0}}\left(\Upsilon_{T}^{u}\left(\psi_{1}\right)\right)=\Pi_{n_{0}}\left(\psi_{2}\right)
$$

Lemma 12 guarantees the existence of a neighborhood $\mathcal{N} \subset G(X)$ of $\Pi_{n_{0}}\left(e^{\gamma A} \psi_{2}\right)$. By Lemma 9 with $\mu=\gamma$, there exists $\tau \geq 0$ and $\zeta \in \mathcal{N}$ such that $e^{\tau} \zeta=\Pi_{n_{0}}\left(\psi_{2}\right)$. Let $s \in X$ be such that $\Pi_{n_{0}}\left(\Upsilon_{T_{s}}^{v^{s}}\left(\psi_{1}\right)\right)=\zeta$. The concatenation of $v^{s}$ and the zero function for a time $\tau$ then steers $\psi_{1}$ to some $\psi_{3}$ such that $\Pi_{n_{0}} \psi_{3}=\Pi_{n_{0}} \psi_{2}$. Notice that such a concatenation is admissible since, in the case in which $\mathcal{U}$ is the set of $C^{\infty}$ functions with values in $U$, the control $v^{s}$, given by Lemma 12 (see also Proposition 8), is zero with all derivatives at $T_{s}$.

\section{References}

[1] R. Adami and U. Boscain. Controllability of the Schrödinger equation via intersection of eigenvalues. In Proceedings of the 44th IEEE Conference on Decision and Control, pages 1080-1085, 2005 .

[2] A. A. Agrachev and M. Caponigro. Dynamics control by a time-varying feedback. J. Dyn. Control Syst., 16(2):149-162, 2010.

[3] A. A. Agrachev and Y. L. Sachkov. Control theory from the geometric viewpoint, volume 87 of Encyclopaedia of Mathematical Sciences. Springer-Verlag, Berlin, 2004. Control Theory and Optimization, II. 
[4] J. M. Ball, J. E. Marsden, and M. Slemrod. Controllability for distributed bilinear systems. SIAM J. Control Optim., 20(4):575-597, 1982.

[5] K. Beauchard and J.-M. Coron. Controllability of a quantum particle in a moving potential well. J. Funct. Anal., 232(2):328-389, 2006.

[6] K. Beauchard and C. Laurent. Local controllability of 1D linear and nonlinear Schrödinger equations with bilinear control. J. Math. Pures Appl., 94(5):520-554, 2010.

[7] R. S. Bliss and D. Burgarth. Quantum control of infinite-dimensional many-body systems. Phys. Rev. A, 89:032309, Mar 2014.

[8] A. M. Bloch, R. W. Brockett, and C. Rangan. Finite controllability of infinite-dimensional quantum systems. IEEE Trans. Automat. Control, 55(8):1797-1805, 2010.

[9] U. Boscain, M. Caponigro, T. Chambrion, and M. Sigalotti. A weak spectral condition for the controllability of the bilinear schrödinger equation with application to the control of a rotating planar molecule. Communications in Mathematical Physics, 311(2):423-455, 2012.

[10] U. Boscain, M. Caponigro, and M. Sigalotti. Multi-input Schrödinger equation: Controllability, tracking, and application to the quantum angular momentum. Journal of Differential Equations, 256(11):3524 - 3551, 2014.

[11] U. Boscain, F. Chittaro, P. Mason, and M. Sigalotti. Adiabatic control of the Schroedinger equation via conical intersections of the eigenvalues. IEEE Trans. Automat. Control, 57(8):19701983, 2012.

[12] N. Boussaïd, M. Caponigro, and T. Chambrion. Regular propagators of bilinear quantum systems. Preprint arXiv:1406.7847, 2016.

[13] T. Chambrion. Periodic excitations of bilinear quantum systems. Automatica J. IFAC, 48(9):2040-2046, 2012.

[14] T. Chambrion, P. Mason, M. Sigalotti, and U. Boscain. Controllability of the discrete-spectrum Schrödinger equation driven by an external field. Ann. Inst. H. Poincaré Anal. Non Linéaire, 26(1):329-349, 2009.

[15] D. D'Alessandro. Introduction to quantum control and dynamics. Applied Mathematics and Nonlinear Science Series. Boca Raton, FL: Chapman, Hall/CRC., 2008.

[16] R. El Assoudi, J. P. Gauthier, and I. A. K. Kupka. On subsemigroups of semisimple Lie groups. Ann. Inst. H. Poincaré Anal. Non Linéaire, 13(1):117-133, 1996.

[17] J.-P. Gauthier and G. Bornard. Controlabilité des systèmes bilinéaires. SIAM J. Control Optim., 20(3):377-384, 1982.

[18] O. Glass and L. Rosier. On the control of the motion of a boat. Math. Models Methods Appl. Sci., 23(4):617-670, 2013.

[19] M. Herrero-Collantes and J. C. Garcia-Escartin. Quantum random number generators. Rev. Mod. Phys., 89:015004, Feb 2017. 
[20] V. Jurdjevic and I. Kupka. Control systems on semisimple Lie groups and their homogeneous spaces. Ann. Inst. Fourier (Grenoble), 31(4):vi, 151-179, 1981.

[21] V. Jurdjevic and H. J. Sussmann. Control systems on Lie groups. J. Differential Equations, 12:313-329, 1972.

[22] M. Keyl, R. Zeier, and T. Schulte-Herbrueggen. Controlling Several Atoms in a Cavity. New J. Phys., 16:065010, 2014.

[23] X. Ma, X. Yuan, Z. Cao, B. Qi, and Z. Zhang. Quantum random number generation. npj Quantum Information, 2:16021, 2016.

[24] P. Mason and M. Sigalotti. Generic controllability properties for the bilinear Schrödinger equation. Comm. Partial Differential Equations, 35(4):685-706, 2010.

[25] M. Mirrahimi. Lyapunov control of a quantum particle in a decaying potential. Ann. Inst. H. Poincaré Anal. Non Linéaire, 26(5):1743-1765, 2009.

[26] M. Mirrahimi and P. Rouchon. Controllability of quantum harmonic oscillators. IEEE Transactions on Automatic Control, 49(5):745-747, 2004.

[27] M. Morancey and V. Nersesyan. Simultaneous global exact controllability of an arbitrary number of 1D bilinear Schrödinger equations. J. Math. Pures Appl. (9), 103(1):228-254, 2015.

[28] V. Nersesyan. Growth of Sobolev norms and controllability of the Schrödinger equation. Comm. Math. Phys., 290(1):371-387, 2009.

[29] V. Nersesyan. Global approximate controllability for Schrödinger equation in higher Sobolev norms and applications. Ann. Inst. H. Poincaré Anal. Non Linéaire, 27(3):901-915, 2010.

[30] V. Nersesyan and H. Nersisyan. Global exact controllability in infinite time of Schrödinger equation. J. Math. Pures Appl. (9), 97(4):295-317, 2012.

[31] E. Paduro and M. Sigalotti. Approximate controllability of the two trapped ions system. Quantum Inf. Process., 14(7):2397-2418, 2015.

[32] M. Reed and B. Simon. II: Fourier Analysis, Self-Adjointness, volume 2. Elsevier, 1975.

[33] S. G. Schirmer, H. Fu, and A. I. Solomon. Complete controllability of quantum systems. Physical Review A, 63(6):063410, 2001.

[34] H. J. Sussmann. Some properties of vector field systems that are not altered by small perturbations. J. Differential Equations, 20(2):292-315, 1976.

[35] G. Turinici. On the controllability of bilinear quantum systems. In M. Defranceschi and C. Le Bris, editors, Mathematical models and methods for ab initio Quantum Chemistry, volume 74 of Lecture Notes in Chemistry. Springer, 2000. 